\title{
Ultrasound Mapping of Stimulated Finger Flexion During Infra-Clavicular Brachial Plexus Nerve Blockade for Elbow Arthroplasty and Its Correlation To Postoperative Analgesia
}

\author{
Case Report
}

L. Lollo*, A. Stogicza

Department of Anesthesiology and Pain Medicine, University of Washington, Seattle, WA, USA.

\section{Abstract}

Background: Infraclavicular brachial plexus nerve blockade (ICNB) is a very common anesthetic procedure performed for upper extremity surgery at the elbow and distally, however the rate of adequate analgesia is variable among patients. Ultrasound guidance (US) has not been demonstrated to increase the success rate of ICNB when compared to nerve stimulator (NS) guidance. Combined US and NS guided ICNB have not been reported, although there is a call for more trials comparing the two techniques. This study was performed to observe if a specific anatomic region near the axillary artery of the brachial plexus identified by finger flexion with nerve stimulation results in improved postoperative analgesia.

Method: Patients undergoing elective elbow arthroplasty received a postoperative ICNB. The angle of the nerve stimulator needle tip and the radial distance from the center of the arterial lumen at which an optimal finger flexion twitch response was observed were measured with ultrasound imaging. Pain scores and postoperative opioid dosages on discharge from the post anesthesia care unit and at 24 hours after surgery were recorded.

Results: 11 patients enrolled in this study. Adequate finger flexion response to nerve stimulation that resulted in complete analgesia was more frequently observed when the needle was located in the postero-superior quadrant in relation to the axillary artery. Identifying a specific point near the brachial plexus in relation to the artery that consistently provides superior analgesia is desirable and would lead to improved analgesia and faster onset time of nerve blockade and would reduce the need for other approaches for brachial plexus blockade with their associated disadvantages.

\section{*Corresponding Author:}

\section{Lollo MD,}

Department of Anesthesiology and Pain Medicine, Box 356540, University of Washington, 1959 NE Pacific St, BB-1469, Seattle, WA 981956540, USA.

Tel: 206-744-2881

Fax: 206-7446240

E-mail: lollomd@uw.edu

Received: May 23, 2015

Accepted: June 16, 2015

Published: June 17, 2015

Citation: L. Lollo, A. Stogicza (2015) Ultrasound Mapping of Stimulated Finger Flexion During Infra-Clavicular Brachial Plexus Nerve Blockade for Elbow Arthroplasty and Its Correlation to Postoperative Analgesia. Int J Anesth Res. 3(5), 113-115. doi: http://dx.doi.org/10.19070/23322780-1500029

Copyright: L. Lollo ${ }^{\circ}$ 2015. This is an open-access article distributed under the terms of the Creative Commons Attribution License, which permits unrestricted use, distribution and reproduction in any medium, provided the original author and source are credited.

\section{Introduction}

Brachial plexus nerve blockade for upper extremity surgery is a common anesthetic procedure that has become more widely used with the introduction of ultrasound guided imaging. Infraclavicular brachial plexus nerve blockade (ICNB) is a technique that requires the least time to perform when compared to the other accepted techniques including the interscalene, supraclavicular and axillary approaches. (1) The disadvantage of the ICNB is the slower onset time when compared to the other techniques. (2) The advantages of the ICNB to the axillary approach are the use of a single injection instead of multiple injections to produce brachial plexus nerve blockade and simultaneous anesthesia of the musculocutaneous and axillary nerves. (3) The advantages of the ICNB to the interscalene and supraclavicular approaches are the avoidance of respiratory problems caused by pneumothorax, phrenic nerve blockade, and swallowing difficulty from vagus nerve blockade. (4) The ICNB also demonstrates superior prolonged analgesia compared to the supraclavicular approach. (5) Ultrasound guidance (UG) has not been demonstrated to increase the success rate of ICNB when compared to nerve stimulator (NS) guidance. (6) Distal motor response or finger flexion has been demonstrated to be the appropriate endpoint for NS guided ICNB. (7) Nerve stimulator current causing finger flexion that disappears above $0.6 \mathrm{~mA}$ is associated with poor analgesic response and finger flexion at current below $0.3 \mathrm{~mA}$ is an indicator of intraneural needle tip placement and risks neural injury. (8) The incidence of being unable to identify the brachial plexus by either ultrasound or nerve stimulation has been reported to be $10 \%$ and is postulated to be related to a variety of factors including variant patient anatomy and diabetic neuropathy. (9) Combined US and NS guided ICNB have not been reported, although there is a call for more trials comparing the two techniques. ICNB is a very common anestheticprocedure performed for upper extremity surgery at the elbow and distally, however the rate of adequate analgesia is variable among patients. 
The purpose of this study was to use ultrasound imaging to map the finger flexor response elicited by nerve stimulation during infra-clavicular brachial plexus nerve blockade (ICNB) and correlate local anesthetic injection at this point with postoperative analgesia. The hypothesis tested was if a specific anatomicregion near the axillary artery of the brachial plexus identified by finger flexion with nerve stimulation results in improved postoperative analgesia.

\section{Method}

After receiving IRB approval from the University of Washington Human Subjects Division, patients provided written informed consent prior to undergoing elective elbow arthroplasty and were enrolled for participation in this prospective study of the perioperative analgesic effects of ICNB. Preoperative data collected were age, gender, ASA physical status, height, weight, calculated body mass index (BMI), maximum pain score and dosage of opioid analgesia used in the 24 hours preceding surgery. Exclusion criteria were age below 18 years, pre-existing neuropathy, neurologic disease, recent trauma or other comorbidity altering sensation in the affected extremity and the use of opioids for a period over three months for any pain disorder unrelated to the planned upper extremity surgery. All procedures were performed at Harborview Medical Center, Seattle between January and June, 2012.

All patients received general inhalational endotracheal anesthesia with sevoflurane and intraoperative analgesia in the form of intravenous fentanyl, morphine and/or hydromorphone for their surgery. The dose of intraoperative opioid administered was recorded for each patient.

Postoperative ICNB by the sagittal coracoid approach was performed in the post anesthesia care unit. All patients were administered $30 \mathrm{ml} 0.5 \%(150 \mathrm{mg})$ ropivacaine for the ICNB using a Life-Tech ProBloc II 20 Gauge 50mm 30 degree bevel needle. The procedure was performed with the use a Life-Tech Tracer III nerve stimulator (NS) under ultrasound guidance (USG) using a SonoSite M Turbo with a linear $38 \mathrm{~mm}$ probe to locate the axillary artery in the short axis view and the dose of local anesthetic was injected when finger flexion was observed at a current of less than $0.6 \mathrm{~mA}$. The angle of the nerve stimulator needle tip in relation to the anterior sagittal plane of the axillary artery and the radial distance from the center of the arterial lumen at which the minimal current above $0.3 \mathrm{~mA}$ produced an optimal finger flexion twitch response as assessed by two regional anesthesia faculty observers were measured and the injection point was plotted on a reference compass drawing of the subclavian artery for each patient. All the patient measurements were plotted as a scattergram of the needle tip coordinates at which the optimal twitch response in relation to the anatomic location of the axillary artery were achieved. The zero reference point for measurement was the perpendicular drawn from the center of the axillary artery lumen to the anterior chest wall surface. The nerve block procedures were supervised by regional anesthesia faculty with added expertise in ultrasound guided imaging.

Patient self-reported pain scores, observation of voluntary finger flexion, and total postoperative opioid doses converted to $\mathrm{mg}$ of intravenous MS on discharge from the post anesthesia care unit (PACU) and at 24 hours after surgery were recorded.

Postoperative analgesia in those patients with inadequate pain relief following ICNB was administered as intravenous fentanyl, morphine and/or hydromorphone and oral oxycodone in bolus doses and patient controlled analgesic infusions of morphine, fentanyl or hydromorphonein the first 24 hours following surgery. Intravenous patient controlled analgesia (PCA) using hydromorphone was prescribed to all patients for relief of breakthrough pain at a rate of $0.2 \mathrm{mg}$ every 6 minutes with an escalation dose of $0.4 \mathrm{mg}$ without a 6 hour maximum dose lockout restriction.

The variety of analgesic narcotic medications administered perioperatively due to patient and prescribing practitioner preferences required the conversion to an equipotent unit in order to allow comparison of dosages and these values were calculated as $\mathrm{mg}$ of intravenous morphine sulphate using standardized opioid conversion formulae.

\section{Results}

Measurements were recorded for 11 patients during this study period and their preoperative demographics are summarized in Table 1 . The perioperative pain scores and opioid dosages for the 24 hour period of observation and measurement are also presented in the same Table.

Figure 1 is the plot of the coordinates of the nerve block needle tip at which finger flexion was observed for each patient and these points are coded in relation to their post-operative care unit discharge pain scores. Analgesia was recorded as either complete with pain score 0 , partial with pain score 2 to 3 , and incomplete with pain scores over 5 . All patients retained voluntary finger flex-

Table 1. Patient peri-operative demographics.

\begin{tabular}{|c|c|c|}
\hline $\mathbf{N}=\mathbf{1 1}$ & Female (N = 5) & Male (N=6) \\
\hline Age $(\mathrm{yrs})$ & $57.2(7.5)$ & $43.3(14.9)$ \\
\hline BMI $\left(\mathrm{kg} / \mathrm{m}^{2}\right)$ & $30.5(9.5)$ & $28.5(3.3)$ \\
\hline ASA & 2 & $2(1)$ \\
\hline Preop Pain Score & $2(3)$ & $3(4)$ \\
\hline Preoperative Opoids & $7(13)$ & $7(12)$ \\
\hline Intraoperative Opioids & $24.5(17)$ & $28.8(9.5)$ \\
\hline PACU Pain Score & $2(4)$ & $2(1)$ \\
\hline PACU Opioids & $5.6(8.2)$ & $6.8(9.7)$ \\
\hline 24 Hour Pain Score & $5(3)$ & $6(2)$ \\
\hline 24 Hour Opioids & $86.1(94.5)$ & $93.5(71.4)$ \\
\hline
\end{tabular}


Figure 1. Coordinate diagram for needle position in relation to subclavian artery at which finger flexion was elicited by nerve stimulation at 0.3 to $0.6 \mathrm{~mA}$. Green stars indicate pain score 0 in PACU. Yellow circles indicate pain score $2-3$ in PACU, and red cross indicates pain score over 5 .

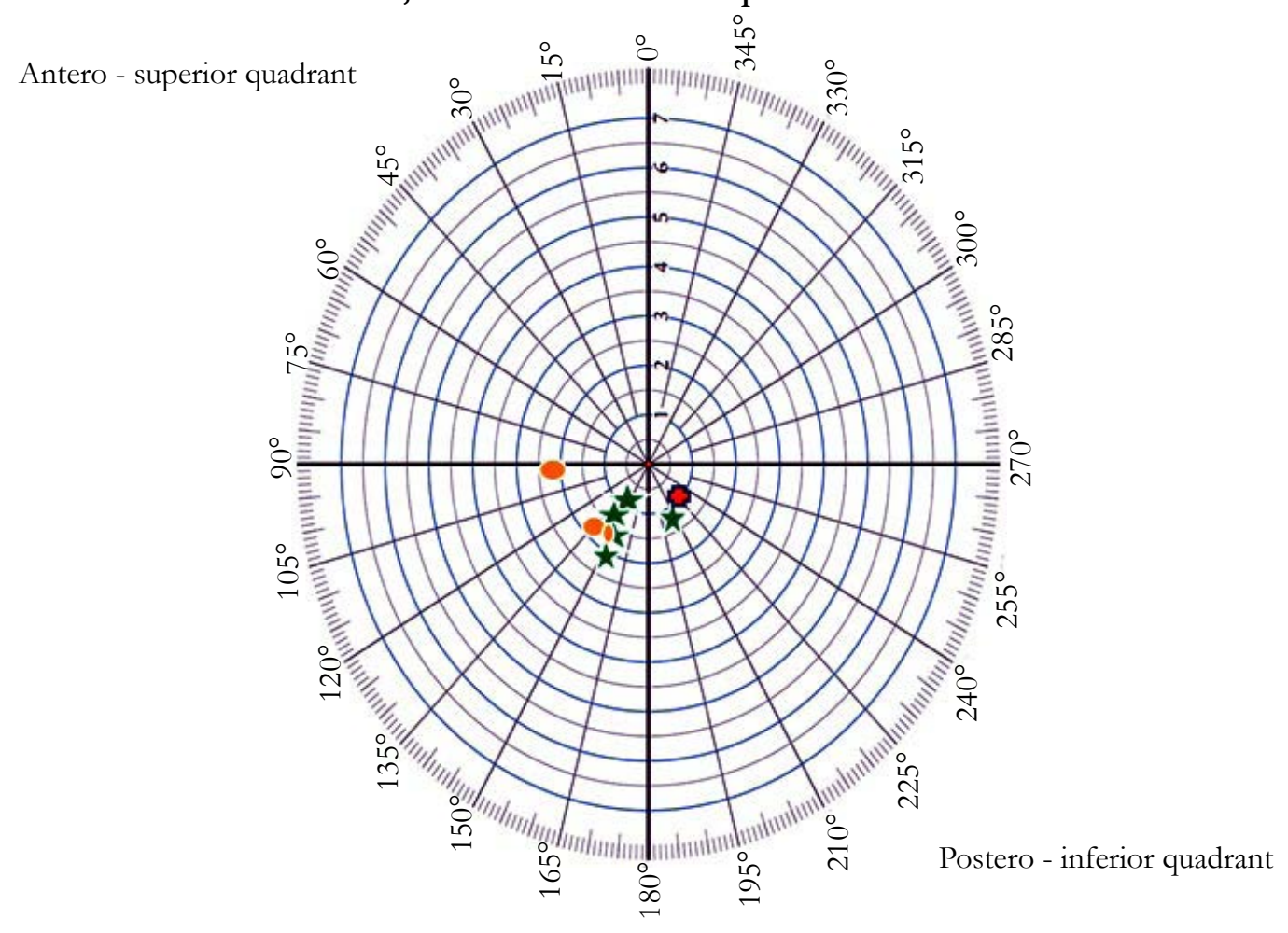

ion after the nerve block was administered.

\section{Discussion}

The needle coordinates that demonstrated adequate finger flexion response to nerve stimulation and that resulted in complete analgesia from ICNB was more frequently located in the posterosuperior $(-\mathrm{x},-\mathrm{y})$ quadrant at a point 150 degrees from the anterior sagittal plane. The reference point for the measurements is the $(-\mathrm{x}$, $+y)$ quadrant that corresponds to the antero-superior region of the axillary artery closest to the skin and clavicle. In one patient the distance from the arterial lumen was $7 \mathrm{~mm}$ at 225 degrees but inadequate ICNB resulted.

The small number of patients enrolled precluded statistical analysis or generalizations and more study into this observation is warranted.

Identifying a specific point near the brachial plexus in relation to the axillary artery that consistently provides superior analgesia is desirable and would lead to improved outcomes with regard to postoperative pain scores. Identification of this location is possible by combined nerve stimulation assisted with ultrasound mapping and knowledge of this particular anatomic location of the brachial plexus in the infra-clavicular region associated with improved analgesia and faster onset time of nerve blockade would reduce the need for other approaches for brachial plexus blockade with their associated disadvantages.

Future studies point to further mapping of the three dimensional diffusion of local anesthetic in the area surrounding peripheral nerves and identifying if the perineural or adjacent fascial space is related to improved analgesia. The real time three dimensional display of the diffusion of local anesthetic in the perineural region has recently become possible with improved ultrasound imaging technology. The long term impact of this study is that ultrasound only based nerve blockade will be more accurate and eliminate the painful nerve stimulator adjunct currently used in practice.

The authors report no external funding source for this study and also report no declarations of interest.

\section{References}

[1]. Song IA, Gil NS, Choi EY, Sim SE, Min SW, et al. (2011) Axillary approach versus the infraclavicular approach in ultrasound-guided brachial plexus block: comparison of anesthetic time. Korean J Anesthesiol 61(1): 12-18.

[2]. Chin KJ, Singh M, Velayutham V, Chee V (2010) Infraclavicular brachial plexus block for regional anaesthesia of the lower arm. Anesth Analg 111(4): 1072 .

[3]. Mariano ER, Sandhu NS, Loland VJ, Bishop ML, Madison SJ, et al. (2011) A randomized comparison of infraclavicular and supraclavicular continuous peripheral nerve blocks for postoperative analgesia. Reg Anesth Pain Med 36(1): 26-31.

[4]. Gelfand HJ, Ouanes JP, Lesley MR, Ko PS, Murphy JD, et al. (2011) Analgesic efficacy of ultrasound-guided regional anesthesia: a meta-analysis. J Clin Anesth 23(2): 90-96.

[5]. Lang RS, Kentor ML, Vallejo M (2012) The impact of local anesthetic distribution on block onset in ultrasound guided interscalene nerve block. ActaAneaesthesiol Scand 56 (9): 1146 - 1151. 